\title{
Clinical Diagnostic Value of Combined Detection of Serum C-Reactive Protein and Procalcitonin for Bacterial Infectious Diseases in Children
}

\author{
Xin Ge, Peng Li and Zhigang Wu
}

\begin{abstract}
The aim of this study was to investigate the clinical diagnostic value of combined detection of serum C-reactive protein (CRP) and procalcitonin (PCT) for bacterial infectious diseases in children. It was a case control study carried out from October 2016 to March 2018. A total of 150 children with bacterial infectious disease were assigned to the observation group, and 150 healthy children without infectious diseases were assigned to the control group. The research showed that CRP and PCT levels, positive rates of CRP and PCT detection in observation group were higher than those in control group $(p<0.001)$; sensitivity and diagnostic coincidence rate, detection specificity of CRP combined with PCT were higher than those in single PCT or CRP ( $<<0.001,<0.001$ and 0.013 , respectively). CRP combined with PCT detection enjoys good clinical application value in diagnosis of bacterial infectious diseases in children.
\end{abstract}

Key Words: C-reactive protein, Procalcitonin, Bacterial infection, Children.

Bacterial infectious diseases are diseases in which pathogenic bacteria or conditional pathogens invade the blood circulation, produce toxins and cause systemic infections through growth and reproduction. ${ }^{1}$ Bacterial infectious diseases in children is difficult to diagnose as the disease simply forms symptoms and signs of the children and three routine items (blood, urine and excrement), which brings about great difficulties in judging whether antibiotics should be used and which antibiotics to choose. Although the culture of secretions is of great help clinically in the identification of pathogens and drug susceptibility, its detection rate is significantly reduced due to the abuse of antibiotics. Moreover, results are not available in time in case of secretion culture, which is not conducive to instruction on correct use of antibiotics in early stage. Therefore, it is particularly important to find sensitivity inspection indicators early. C-reactive protein (CRP) is an acute phase reaction protein synthesised by the liver when the body tissue is damaged. ${ }^{2}$ Procalcitonin (PCT) secreted by thyroid $\mathrm{C}$ cells under physiological conditions is selectively expressed on neuroendocrine cells of thyroid and lungs, which cannot be easily detected due to the low circulating concentration. In pathological conditions, PCT is secreted by parenchymal cells of various organs such as liver, kidney, fat, and muscle, which can be easily detected due to the significantly increased circulating concentration. ${ }^{3}$

Maternity and Child Care Center of Qinhuangdao,

Qinhuangdao, 066000, China

Correspondence: Xin Ge, Maternity and Child Care Center of

Qinhuangdao, Qinhuangdao, 066000, China

E-mail:frv7ll@163.com

Received: May 08, 2018; Accepted: September 09, 2018
The aim of this study was to investigate the clinical diagnostic value of combined detection of serum CRP and PCT for bacterial infectious diseases in children.

This case-control study was carried out at the Maternity \& Child Care Center of Qinhuangdao, China, from October 2016 to March 2018. A total of 150 children with bacterial infectious disease were assigned to an observation group. Observation group met the diagnostic criteria for bacterial infectious diseases of American College of Chest Physicians and Consensus Conference of Society of Critical Care Medicine. Bacterial infectious diseases in children included pneumonia, enteritis, meningitis, urinary system diseases, etc. Children with tumors, severe trauma, and cardiogenic shock were excluded. One hundred and fifty healthy children without infectious diseases for the same period were assigned to a control group.

Forearm venous blood was withdrawn. CRP was measured by immunoturbidimetric assay, and CRP value $\geq 8 \mathrm{mg} / \mathrm{L}$ was positive. The PCT was measured by double antibody sandwich immuno-luminescence assay, and PCT value $\geq 0.5 \mathrm{ng} / \mathrm{mL}$ was positive. The positive rates of PCT and CRP in two groups were compared. The sensitivity and diagnostic coincidence rates were comparatively detected by PCT and CRP and PCT or CRP alone. Collected data were analysed using independent sample t-test and Chi-square test by Statistical Package for Social Sciences (SPSS) version 21. $\mathrm{P}$-value of less than 0.05 was considered significant.

The observation group included 80 males (53.33\%) and 70 females (46.67\%); aged 8 months to 12 years, with an average age of $5.21 \pm 1.75$ years; had $90(60.00 \%)$ cases of bacterial pneumonia, $14(9.33 \%)$ cases of purulent meningitis, $40(26.67 \%)$ cases of suppurative 
Table I: Comparison of CRP and PCT diagnostic rates.

\begin{tabular}{|c|c|c|c|c|c|c|}
\hline \multirow[t]{2}{*}{ Testing index } & \multicolumn{2}{|c|}{ Detection sensitivity $(n=150)$} & \multicolumn{2}{|c|}{ Detection specificity $(n=150)$} & \multicolumn{2}{|c|}{ Diagnostic coincidence rate $(n=300)$} \\
\hline & $\mathrm{n}(\%)$ & $p$-value & $\mathrm{n}(\%)$ & $p$-value & $n(\%)$ & $p$-value \\
\hline Single CRP & $112(74.67)$ & $<0.001$ & $137(91.33)$ & 0.013 & $249(83.00)$ & $<0.001$ \\
\hline Single PCT & $113(75.33)$ & & $138(92.00)$ & & $251(83.67)$ & \\
\hline CRP combined with PCT & 149 (99.33) & & $148(98.67)$ & & $297(99.00)$ & \\
\hline
\end{tabular}

tonsillitis, and $6(4.00 \%)$ cases of sepsis. The control group included 79 males $(52.67 \%)$ and 71 females (47.33\%); aged 7 months to 12 years, with an average age of $5.35 \pm 1.66$ years.

Serum CRP and PCT levels in the observation group were $14.12 \pm 2.20 \mathrm{mg} / \mathrm{L}, 3.09 \pm 0.48 \mathrm{ng} / \mathrm{mL}$, respectively, which were higher than $5.06 \pm 0.73 \mathrm{mg} / \mathrm{L}$ and $0.24 \pm 0.04$ $\mathrm{ng} / \mathrm{mL}$ of the control group $(\mathrm{p}<0.001)$.

Positive rate of CRP and PCT detections in the observation group were 132 cases $(88.00 \%), 135$ cases $(90.00 \%)$, respectively, which were higher than 54 cases $(36.00 \%)$ and 56 cases $(37.33 \%)$ of the control group $(p<0.001)$.

The detection sensitivity and diagnostic coincidence rate of CRP combined with PCT are higher than that of single CRP or PCT, with statistically significant difference $(p<0.001)$; the detection specificity of CRP combined with PCT is higher than that of single CRP or PCT $(p=0.013$, Table I).

Traditionally, the diagnosis of bacterial infectious diseases depends on the number of leukocytes in the blood. The total number of leukocytes in the body is affected by factors such as diet, exercise, and drinking, so the positive diagnostic accuracy is low. Studies have shown the presence of CRP in patients with bacterial infectious disease, which can precipitate in precipitin reaction with pneumoniae C-polysaccharides; CRP levels in patients are significantly elevated under the direct action of inflammatory factors. ${ }^{4}$ PCT can be used to assess the progress of bacterial infectious disease in patients. ${ }^{5}$

This study showed that CRP and PCT levels were higher in the observation group than in the control group. This is because inflammatory factors produced during the bacterial infection and endotoxin produced by the bacteria will stimulate the body, resulting in elevated CRP and PCT concentrations. The positive rate of CRP and PCT were higher in observation group than those in control group, and sensitivity, specificity and diagnostic coincidence rate of the combined detection were higher than those of single CRP or PCT.

To conclude, CRP combined with PCT can effectively diagnose bacterial infectious diseases in children, and practically reflect the condition of bacterial infectious disease in children.

\section{REFERENCES}

1. Zuwala-Jagiello J, Simon K, Kukla M, Murawska-Cialowicz E, Gorka-Dynysiewicz J, Grzebyk E, et al. Increased circulating endocan in patients with cirrhosis: relation to bacterial infection and severity of disease. J Physiol Pharmacol 2017; 68:273-82.

2. Barle $\mathrm{H}$, Hammarqvist $F$, Westman $B$, Klaude $M$, Rooyackers $O$, Garlick PJ, et al. Synthesis rates of total liver protein and albumin are both increased in patients with an acute inflammatory response. Clin Sci (Lond) 2006; 110:93-9.

3. Kowalska A, Lizis-Kolus K, Gasior-Perczak D, Palyga I, Sluszniak A, Sluszniak J, et al. Comparative assessment of calcitonin stimulation test using calcium gluconate and pentagastrin and the usefulness of procalcitonin basic and post-stimulation concentrations in the diagnosis of patients after surgery for medullary. Neuro Endocrinol Lett 2016; 37: 485-90.

4. Meidani M, Khorvash F, Abolghasemi H, Jamali B. Procalcitonin and quantitative c-reactive protein role in the early diagnosis of sepsis in patients with febrile neutropenia. South Asian $J$ Cancer 2013; 2:216-9.

5. Pierce R, Bigham MT, Giuliano JS Jr. Use of procalcitonin for the prediction and treatment of acute bacterial infection in children. Curr Opin Pediatr 2014; 26:292-8. 